\title{
Constitutional Mismatch Repair Deficiency Syndrome
}

National Cancer Institute

\section{Source}

National Cancer Institute. Constitutional Mismatch Repair Deficiency Syndrome. NCI

Thesaurus. Code C130202.

A rare childhood cancer predisposition syndrome caused by biallelic inheritance of mutations in MLH1, MSH2, MSH6, or PMS2 genes. It is characterized by the development of childhood cancers, usually hematological malignancies and/or brain tumors, and colorectal cancers with multiple intestinal polyps. The majority of patients show signs of neurofibromatosis type 1. 